Acta bot. bras. 1(2):221-230 (1988) supl.

\title{
A VEGETAÇÃo ARBÓREA DO PARQUE ESTADUAL DO MORRO DO DIABO, MUNICÍPIO DE TEODORO SAMPAIO, ESTADO DE SÃO PAULO.
}

\author{
João Batista Baitello (1) \\ João Aurélio Pastore (1) \\ Osny Tadeu de Aguiar (1) \\ Francisco Corrêa Sério (1) \\ Carlos Eduardo F. da Silva (1)
}

\begin{abstract}
RESUMO - Fez-se o levantamento florístico da vegetação arbórea da floresta mesófila do Parque Estadual do Morro do Diabo, Município de Teodoro Sampaio, Estado de São Paulo (2230'S, $\left.52^{\circ} 20^{\prime} \mathrm{W}\right)$ pelos métodos de quadrantes e parcelas, incluindo as árvores com diâmetro à altura do peito igual ou superior a $10 \mathrm{~cm}$. A utilizaçâo dos dois métodos deu-se em função das características fisionômicas, solo e drenagem dos locais amostrados. O método de quadrante envolveu 462 pontos com intervalos de $30 \mathrm{~m}$., e o método de parcelas ca. $26.900 \mathrm{~m}^{2}$ (ca. 2,7 ha.). Os parâmetros fitossociológicos serão abordados em futuros trabalhos. Os dados florísticos subsidiaram o reassentamento dos grupos faunísticos, em especial o Leontopithecus chrysopygus Mikan, 1823 (mico-leão-preto), dentro das áreas remanescentes. $\mathrm{O}$ "check list" inclui também algumas espécies coletadas aleatoriamente em outras áreas do Parque. Constatou-se nas áreas de amostragem e adjacentes 113 espécies, 95 gêneros e 42 famílias, das quais 6 contribuíram com $56 \%$ das espécies levantadas. As famílias mais representativas no Parque, envolvendo todos os locais de coleta são: Leguminosae (13 Faboideae, 6 Caesalpinioideae e 6 Mimosoideae), Rutaceae 11, Meliaceae 8, Lauraceae 7, Euphorbiaceae 7 e Myrtaceae 6. A listagem das espécies revela que o Parque Estadual do Morro do Diabó apresenta uma vegetação de grande heterogeneidade florística.
\end{abstract}

ABSTRACT - In a mesophyll forest at the State Park of "Morro do Diabo", in the municipality of Teodoro Sampaio, State of São Paulo ( $\left.22^{\circ} 30^{\prime} \mathrm{S}, 52^{\circ} 20^{\prime} \mathrm{W}\right)$ the floristic composition was surveyed. The point centered quarter and quadrant methods were used to survey trees with a diameter equal or greater than $10 \mathrm{~cm}$ at breast height. These methods were used as a function of soil features, drainage and physionomical characteristics of the place where the samples were taken. The quarter method sampled 462 points with intervals of $30 \mathrm{~m}$, and the other one had an area of approximately $26.900 \mathrm{~m}^{2}$. The phytosociological parameters will be reported in a future paper. The floristic data were important to the resettlement of faunistic groups, particulary Leontopithecus chrysopygus Mikan, 1823 ("mico-leão-preto") within remaining areas. The check list also included some species colected in other areas of the Park. There were verified in the sampling areas and surrondings, 113 species, 95 genera and 42 families of trees. Six (6) families contributed with $56 \%$ of the total number of species. The most representative families in the Park were the following: Leguminosae 25 (13 Faboideae, 6 Caesalpinioideae and 6 Mimosoideae), Rutaceae 11, Meliaceae 8, Lauraceae 7, Euphorbiaceae 7 and Myrtaceae 6. The checklist showed that the State Park of "Morro do Diabo" has a great floristic heterogeneity.

Key-words: forest, floristic composition, State Park of "Morro do Diabo".

\section{Introdução}

O Parque Estadual do Morro do Diabo foi criado originalmente como Reserva Estadual e contava com 37.156,68 ha. de área (Campos \& Heinsdijk 1970). Face à necessidade de construção do reservatório para a Usina Hidrelétrica de Rosana, da Companhia Energética de São Paulo, foram desapropriados 3.000 ha. às margens do rio Paranapanema.

(1) Instituto Florestal de São Paulo - C.P. 1322 - CEP 01051 - São Paulo - SP. 
Transformada em Parque Estadual, a área conta atualmente com 34.156,68 ha. (SÃO PAULO, Leis, decretos, etc. 1986). Parte dessa área é coberta por mata de planalto com interfaces com cerradão. Segundo IBGE (1970), a vegetação original da região é enquadrada na Formação Floresta Semi-Úmida do interior.

A topografia, levemente ondulada, é quebrada com a presença da elevação denominada "Morro do Diabo" que alcança $300 \mathrm{~m}$. acima do nivel das águas adjacentes (Deshler 1975). Segundo Ventura et al. (1965-66), predomina o solo tipo latossolo vermelho-escuro, fase arenosa (LEa). Referem ainda que o clima é quente de inverno seco (Cwa, na classificação de Köppen), com precipitação média anual de $1130 \mathrm{~mm}$.

Campos \& Heinsdijk (1970) consideraram a vegetação da então Reserva Estadual como um dos últimos remanescentes florestais do planalto ocidental do Estado de São Paulo. Para a elaboração do plano de manejo, inventariaram a vegetação, classificando-a em 6 (seis) tipos arbóreos e 1 (hum) herbáceo-arbustivo. A identificação das espécies florestais envolvidas no inventário deu-se com base unicamente no lenho, o que resultou numa listagem apenas aproximada das faminias e gêneros locais (Mainieri 1970).

O levantamento florístico ora apresentado, com base em parâmetros fitossociológicos, possibilitou um conhecimento mais amplo da vegetação arbórea, fornecendo subsidios aos trabalhos de reassentamento de grupos faunisticos que por força da inundação, em parte da área estudada, foram deslocados para novas áreas floristicamente semelhantes.

\section{Material e Métodos}

A área estudada localiza-se no Município de Teodoro Sampaio, Estado de São Paulo, nas coordenadas $22^{\circ} 30^{\prime}$ lat. Sul e $52^{\circ} 20^{\prime}$ long. Oeste de Greenwich (Fig. 1).

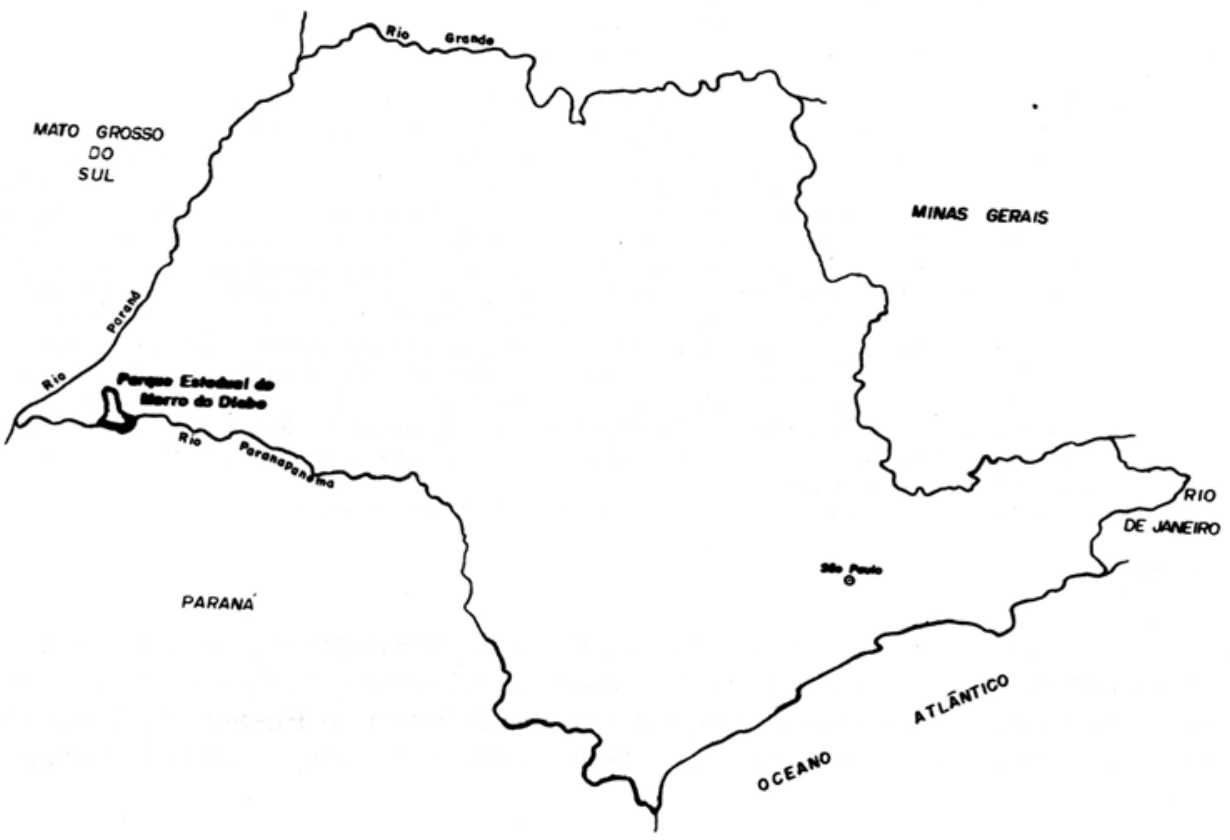

Fig. 1 - Localização do Parque Estadual do Morro do Diabo no Estado de Săo Paulo. 
O projeto foi desenvolvido nos 3.000 ha. da então Reserva Estadual do Morro do Diabo, cujos restantes 34.156,68 ha. hoje compreendem o Parque Estadual do Morro do Diabo. Trata-se de uma faixa marginal do rio Paranapanema, que abrangeu aquela Unidade, conforme outorgado à Companhia Energética de São Paulo, através do Decreto Federal $n=81.689$, de 19 de maio de 1978. Tal concessão deu-se para o aproveitamento de energia hidráulica de trechos daquele rio, no caso a construção do Reservatório Hidrelétrico de Rosana. A legislação vigente determina a destoca e consequente limpeza das bacias hidráulicas artificais, razão pela qual foi considerado prioritário o início dos estudos na faixa de 3.000 ha.

Foram instaladas 10 (dez) áreas de amostragem fitossociológica, cuja localização baseou-se na fitofisionomia e na existência de espécies de fauna em extinção como o mico-leão-preto (Leontopithecus chrysopygus Mikan, 1823).

Face ao período extremamente curto para levantamento da vegetação, tendo em vista o desmatamento e posterior inundação, utilizou-se os métodos de distâncias e de parcelas, conforme o tipo do material vegetal a ser levantado (mata densa, mata rala ou mata com sub-bosque denso) e das facilidades de instalação (terreno firme, terreno úmido ou terreno recoberto por duna lodosa). Assim, 4 (quatro) áreas de amostragem foram levantadas por parcelas e 6 (seis) por quadrantes.

Em ambos os casos utilizou-se como diâmetro mínimo à altura do peito (DAP) o valor de $10 \mathrm{~cm}$. Cada indivíduo recebeu um número, sendo anotado a denominação vulgar regional e mensurados os parâmetros dendrométricos (altura e diâmetro).

$\mathrm{Na}$ utilização do método de quadrantes, a determinação da distância entre pontos de amostragem, deu-se a partir da metodologia descrita por Silva (1980). Foram medidas 100 (cem) distâncias entre árvores mais próximas. A distância máxima encontrada foi dobrada, optando-se por um valor final de $30 \mathrm{~m}$. Em cada ponto foram plaqueadas, por quadrante, os indivíduos mais próximos com D.A.P. maior ou igual a $10 \mathrm{~cm}$., correspondendo, portanto a 4 (quatro) árvores. O número de pontos por área de amostragem é apresentado a seguir:

A área de amostragem 4 com 78 pontos, foi instalada a montante de cota de inundação do Reservatório, na direção aproximada Leste-Oeste. Este procedimento objetivou o conhecimento do local, que posteriormente foi utilizado para reassentamento de animais capturados na área desmatada. No caso das áreas de amostragem 5-7, aproveitou-se a rede de picadas traçadas na direção Norte-Sul, da cota de inundação do Reservatório até a margem do rio Paranapanema, distanciadas de quilômetro em quilômetro, foram incluidos os seguintes número de pontos; área 5-175 pontos; área 6-73 pontos e área 7-102 pontos. As instalações das áreas de amostragem 9 e 10 não seguiram o mesmo critério, sendo das últimas a serem realizadas, quando então o desmatamento já estava bem adiantado. Portanto, foram traçadas procurando abranger fisionomias ainda pouco levantadas no decorrer do trabalho, e abrangeram os seguintes números de pontos: área 9-19 pontos e área 10-15 pontos. A Figura 2 ilustra a localização das áreas de amostragem, que tiveram um total de 462 número de pontos.

No levantamento por parcelas utilizou-se a forma retangular de $60 \mathrm{~m} \times 75 \mathrm{~m}$ (área de amostragem 1 e 3), outra de $40 \mathrm{~m} \times 100 \mathrm{~m}$ (área de amostragem 8) e uma irregular de $13.899,50 \mathrm{~m}^{2}$ (área de amostragem 2). Esta heterogeneidade se justifica. A parcela de forma irregular é consequência de uma "ilha" de mata residual, circundada por larga extensão de terras recobertas por vegetação graminóide. Foram levantados todos os indivíduos arbóreos existentes nesta porção, com DAP mínimo de $10 \mathrm{~cm}$. Nas áreas de amostragem 1 e 3 utilizou-se a forma de $60 \mathrm{~m} \times 75 \mathrm{~m}$, tendo em vista a facilidade de instalação e acréscimo para o estabelecimento da curva área x espécie. A área de amostragem 8, de $40 \mathrm{~m} \times$ $100 \mathrm{~m}$, é objeto de levantamento preliminar, executado anteriormente, cujos dados foram recuperados e incorporados ao presente trabalho. Todas as parcelas foram subdivididas para fins de análise da curva $\mathrm{n}^{\circ}$ de espécies $\mathrm{x}$ área amostrada e $\mathrm{n}^{\circ}$ de espécies $\times \mathrm{n}^{\circ}$ de parcelas. 


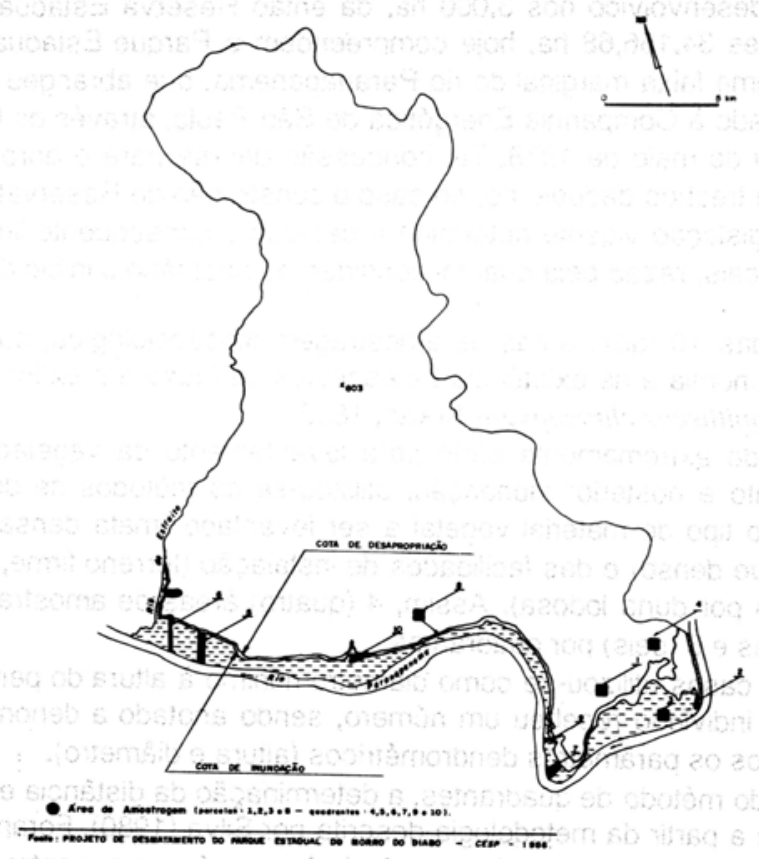

Fig. 2 - Localizaçăo das áreas de amostragem no Parque Estadual do Morro do Diabo.

A análise fitossociológica da vegetação será feita com base na densidade, frequência, dominância e índice de valor de importância.

A utilização de 2 (dois) métodos de levantamento, parcela e quadrante, bem como parcelas de diferentes formas e tamanhos, permitirá uma rica discussão sobre a aplicabilidade dos mesmos, bem como a comparação dos resultados encontrados.

O levantamento florístico foi executado preferencialmente nas áreas de amostragem, estando o material coletado depositado no Herbário D. Bento Pickel (SPSF), do Instituto Florestal, e no Herbário da Companhia Energética de São Paulo (CESP), localizado em Promissão (SP).

A identificação do material botânico foi feita mediante consulta à literatura especializada, em herbários e, através de especialistas.

O material lenhoso foi retirado através de método não destrutivo, com a ajuda de serra, formão e macete, aplicando-se no local da retirada da amostra, solução de $8 \%$ de pentaclorofenol em querozene, evitando-se assim o ataque de organismos xilófagos. As amostras coletadas, num total de 203, estão depositadas na Xiloteca (SPSFw) do Instituto Florestal.

A identificação do material lenhoso foi elaborada a partir do próprio material e também através de cortes histológicos.

\section{Resultados}

O levantamento florístico realizado nas áreas amostradas e, a partir das coletas aleatórias nas demais regiōes do Parque Estadual do Morro do Diabo, revelou grande heterogeneidade de espécies no estrato arbóreo. Quer-se com a inclusão das coletas aleatórias fora da amostragem, representar o maior número possível de espécies. Na ocasião da publicação do tratamento fitossociológico, será feita menção a elas, sem no entanto, quantificá-las.

As espécies encontradas são apresentadas na Tabela 1. 
Tabela 1 - Relação das espécies levantadas no Parque Estadual do Morro do Diabo, Teodoro Sampaio (SP), com suas respectivas famlias e nomes vulgares regionais.

\section{ANACARDIACEAE}

Astronium graveolens Jacq.

Tapirira guianensis Aubl.

guaritá

peito-de-pombo

\section{ANNONACEAE}

Duguetia lanceolata St.-Hil.

pindaiba

Xylopia brasiliensis Spreng.

pimenta-de-macaco

\section{APOCYNACEAE}

Aspidosperma cylindrocarpum Müller Arg.

peroba-poca

Aspidosperma polyneuron Müller Arg.

Peschiera fuchsiaefolia Miers.

peroba-rosa

leiteiro

\section{ARALIACEAE}

Didymopanax morototoni (Aubl.) Decais.

mandioqueira

\section{BIGNONIACEAE}

Tabebuia sp.

Tabebuia avellanedae Lorentz ex Grisebach.

Zeyheria tuberculosa (Vell.) Bur.

ipê-amarelo

ipê-roxo

ipê-tabaco

\section{BOMBACACEAE}

Chorisia speciosa A. St.-Hil.

paineira

\section{BORAGINACEAE}

Cordia ecalyculata Vell.

Cordia trichotoma (Vell.) Arrab.

Patagonula americana $\mathrm{L}$.

canela-preta

louro-pardo

guaiuvira

\section{BURSERACEAE}

Protium heptaphyllym March.

almécega

\section{CARICACEAE}

Jacaratia spinosa (Aubl.) A. DC.

jacaratiá

COMBRETACEAE

Terminalia sp.

amarelinho-do-brejo

Terminalia brasiliensis Eichl. 
cont.

\section{ELAEOCARPACEAE}

Sloanea cf. gracilis Vitt.

Sloanea guianensis Benth.

urucum

\section{ERYTHROXYLACEAE}

Erythroxylum cf. myrsinitis Mart.

\section{EUPHORBIACEAE}

Actinostemon concolor (Spreng.) M. Arg.

roxinho

Alchornea triplinervia (Spreng.) M. Arg.

tapiá

Croton floribundus Spreng.

capixingui

Croton urucurana Bail.

sangue-de-drago

Sapium longifolium M. Arg.

Sebastiana edwalliana Pax. et K. Hoff.

leiteiro

Savia dyctiocarpa Müell. Arg.

roxinho

guaraiúva

\section{FLACOURTIACEAE}

Casearia gossypiosperma Briquet.

Casearia sylvestris $\mathrm{Sw}$.

espeteiro

espeteiro

\section{GUTTIFERAE}

Rheedia gardneriana Tr. et PI.

limãozinho

\section{LAURACEAE}

Cryptocarya moschata Nees.

Endlicheria paniculata (Spreng.) Macbride.

Nectandra megapotamica (Spreng.) Mez.

Nectandra membranacea (Sw) Griseb. ssp. cuspidata (Nees)

Rohwer

Ocotea corymbosa (Meissn.) Mez.

Ocotea diospyrifolia (Meissn.) Mez.

Ocotea elegans Mez.

canela-batalha

canelāo

canelinha

canela-amarela

canela

canela

canelinha-cheirosa

\section{LECYTHIDACEAE}

Cariniana estrellensis (Raddi) O.Ktze

jequitibá-branco

\section{LEGUMINOSAE CAESALPINIOIDEAE}

Apuleia leiocarpa (Vog.) Macbr.

carapa

Cassia ferruginea Schrad.

Copaifera langsdorffii Desf.

canafístula

óleo-de-copaíba

Holocalyx balansae Mich.

alecrim-de-campinas

Hymenaea $\mathrm{sp}$.

Hymenaea sp.

Peltophorum dubium (Spreng.) Taubert.

Senna macranthera (Colled.) I. et B. 
cont.

\section{LEGUMINOSAE FABOIDEAE}

Erythrina crista-galli $\mathrm{L}$.

Lonchocarpus guilleminianus (Tul.) Malme

Lonchocarpus leucanthus Burkart.

Machaerium brasiliensis Vog.

Machaerium nictitans (Vell.) Benth.

Machaerium stipitatum (DC.) Vog.

Myrocarpus frondosus $\mathrm{Fr}$. Allem.

Myroxylon balsamum (L.) Harms

Platypodium elegans Vog.

Poecylanthe parviflora Benth.

Pterodon pubescens Benth.

Pterogyne nitens Tul.

Sweetia fruticosa Spreng.

\section{LEGUMINOSAE MIMOSOIDEAE}

Acacia polyphylla DC.

Anadenanthera falcata (Benth.) Speg.

Enterolobium contortisiliquum (Vell.) Morong.

Inga marginata Willd.

Parapiptadenia rigida (Benth.) Brenan.

Pithecellobium edwallii Hoehne.

\section{MAGNOLIACEAE}

Talauma ovata St.-Hil.

\section{MALVACEAE}

Bastardiopsis densiflora (Hook et Arn.) Hassler.

\section{MELASTOMATACEAE}

Miconia latecrenata (DC.) Naud.

MELIACEAE

Cabralea canjerana (Vell.) Mart. ssp. canjerana

Cedrela fissilis Vell.

Guarea guidonia (L.) Sleumer

Guarea macrophylla Vahl. subsp. tuberculata (Vell.) Penn.

Trichilia casaretti C. DC.

Trichilia catigua A. Juss.

Trichilia elegans A. Juss. subsp. elegans

Trichilia pallida Swartz.

\section{MORACEAE}

Cecropia cf. cinerea Miq.

Chlorophora tinctoria (L.) Gaud.

Ficus enormis (Mart. ex Miq.) Miq.

Sorocea ilicifolium Miq. suinã

feijão-crú

embira-de-sapo

sapuvão

bico-de-pato

sapuva

óleo-pardo

cabreúva

amendoim-do-campo

coração-de-negro

sucupira

amendoim-bravo

guaiçara

monjoleiro

angico-preto

tamboril

angico-vermelho

farinha-seca

baguaçu

algodoeiro

jacatirão, tangará

canjerana

cedro-rosa

amarelinho

marinheiro

embaúba

taiúva

figueira-branca

canxim 
cont.

\section{MYRTACEAE}

Calyptranthes sp.

Campomanesia cf. maschalantha Kiaersk.

Eugenia brasiliensis Lam.

gabiroba

Eugenia cf. punicifolia (H.B.K.) DC.

Myrceugenia ovata Berg.

Myrcia breviramis (Berg.) Legr.

Myrcia rostrata DC.

Myrciaria sp.

pitanga

carvãozinho

cambui

\section{NYCTAGINACEAE}

Boungainvilea arborea Glaziou

flor-roxa

OLACACEAE

Tetrastylidium englerï Schwacke

mandigau

OPILIACEAE

Agonandra brasiliensis Miers.

tàtu

\section{PALMAE}

Syagrus romanzoffiana (Cham.) Glassman.

coqueiro-tupi

PHYTOLACACEAE

Gallesia gorazema (Vell.) Moq.

pau-d'alho

POLYGONACEAE

Ruprechtia laxiflora Meissn.

falso-triplaris

Triplaris surinamensis Cham.

pau-de-formiga

PROTEACEAE

Roupala brasiliensis Klotzsch.

carne-de-vaca

POLYGALACEAE

1 espécie

RHAMNACEAE

Colubrina rufla Reiss

ROSACEAE

Prunus sellowii Koehne

pessegueiro-bravo

RUBIACEAE

Genipa americana $\mathrm{L}$.

genipapo 
cont.

\section{RUTACEAE}

Balfourodendron riedelianum (Engl.) Engl.

Esenbeckia grandiflora Mart.

pau-marfim

Helietha longifoliata Britton

amarelinho

Metrodorea atropurpurea Fisch.

canela-de-cotia

Metrodorea nigra St.-Hill.

chupa-ferro

Pilocarpus pennatifolius Lem.

Pilocarpus spicatus St.-Hil. ssp. spicatus

jaborandi

jaborandi

Zanthoxylum chiloperone Mart.

Zanthoxylum hiemale St.-Hil.

Zanthoxylium cf. regnelianum Engl.

mamica

mamica-de-porca

mamica-de-porca

Zanthoxylum riedelianum Engl.

mamica-de-cadela

\section{SAPINDACEAE}

Allophyllus edulis (St. Hil.) Radlk.

amarelinho

Diatenopteryx sorbifolia Radlk.

correeira

\section{SAPOTACEAE}

Chrysophyllum gonocarpum (Mart. et Eichler) Engl.

guatambú-de-leite

TILACEAE

Luehea divaricata Mart.

açoita-cavalo

VOCHYSIACEAE

Vochysia tucanorum Mart.

pau-de-vinho

ULMACEAE

Trema micrantha (L.) Blume.

pau-pólvora

Da análise da listagem apresentada, constata-se que a familia mais representada na área, incluindo-se a de amostragem e adjacências, é Leguminosae com 25 espécies (13 Faboideae, 6 Caesalpinioideae e 6 Mimosoideae). Desta, a subfamnila Faboideae encerra o maior número de espécies de todas as famnias levantadas. Em seguida estão Rutaceae (11 espécies), Meliaceae (8 espécies), Lauraceae (7 espécies), Euphorbiaceae (7 espécies) e Myrtaceae (6 espécies). No conjunto as familias citadas representam ca. de $56 \%$ do total das espécies incluídas na presente listagem. Tais espécies estão englobadas em 95 gêneros.

\section{Discussão e Conclusões}

Campos \& Heinsdijk (1970) realizaram o inventário florestal do Morro do Diabo com o fim de estabelecer um método de manejo daquela floresta. Inventariaram 0,4 ha. e, das espécies, computou-se o volume da madeira em pé, sem casca. Utilizaram árvores maiores de $25 \mathrm{~cm}$ de diâmetro ao nivel do peito (DAP) e fuste acima de $4 \mathrm{~m}$. Tais parâmetros são mais restritivos que os adotados no presente trabalho. 
Coube a Mainieri (1970) a identificação das espécies, a partir da estrutura do lenho e casca. Segundo o citado autor, o estudo anatômico macro e microscópico pode contribuir para a solução de problemas de identificação mas, a definição da espécie, só poderá ser feita quando comparada com padrōes de lenho classificados botanicamente. Muitas dúvidas acerca da identidade botânica das espécies foram resolvidas, pois coletou-se no presente trabalho, o lenho e o respectivo material botânico. Como era de se esperar, houve um acréscimo de 29 gêneros dos 55 identificados por Mainieri (1970), em vista da menor área de amostragem e de parâmetros mais restritivos estabelecidos por Campos \& Heinsdijk (1970).

A diversidade genérica arbórea da floresta do Morro do Diabo situa-se dentro dos padrōes de heterogeneidade das florestas do Estado de São Paulo, conforme Assumpção et al. (1982).

Os diferentes estudos nas florestas do Estado de Sáo Paulo tem revelado que ao nível de familias e, em certos casos de gêneros, as que apresentam maior riqueza floristica são: Leguminosae, Myrtaceae, Rutaceae, Euphorbiaceae, Lauraceae e Meliaceae.

Variações podem ocorrer, conforme relatam Bertoni et al. (1982), que concluíram que há diferenças entre matas inundáveis e não inundáveis, tanto ao nível de familia quanto ao nível de espécie, em decorrência de comportamentos diferentes conforme o ambiente.

A heterogeneidade florística de nossas matas abriga um patrimônio genético ainda suficiente para qualquer investimento em processos de recuperação de áreas degradadas e estudos da potencialidade silvicultural das espécies florestais.

Tendo em vista que o presente trabalho desenvolveu-se basicamente em área de inundação períodica do rio Paranapanema, seu resultado originou uma listagem de espécies ocorrentes, potencialmente adequadas para o repovoamento das matas ciliares degradadas do Estado de São Paulo.

\section{Referências Bibliográficas}

ASSUMPÇĀO, C.T.; LEITĀO FILHO, H.F. \& CESAR, O. 1982. Descriçāo das matas da Fazenda Barreiro Rico, SP. Revta brasil. Bot., São Paulo, 5(1/2): 53-66.

BERTONI, J.E.A.; STUBBLEBINE, W.H.; MARTINS, F.R. \& LEITĀO FILHO, H.F. 1982. Nota prévia; comparação fitossociológica das principais espécies de florestas de terra firme e de várzea na Reserva Estadual de Porto Ferreira (SP). In: CONGRESSO NACIONAL SOBRE ESSÊNCIAS NATIVAS, Campos do Jordão - SP., set. 12-18, 1982. Anais...Silvic. São Paulo, São Paulo, 16A:563-571. Pt.1 (Ediçāo Especial)

CAMPOS, J.C.C. \& HEINSDIJK, D. 1970. A floresta do Morro do Diabo.Silvic. São Paulo, São Paulo, 7:43-58.

DESHLER, W.O. 1975. Recomendaçōes para o manejo do Morro do Diabo. São Paulo, Instituto Florestal. Public.IF, 6. São Paulo.

INSTITUTO BRASILEIRO DE GEOGRAFIA E ESTATÍSTICA - IBGE. 1970. Mapa da Vegetaçāo. Departamento de Geografia.

MAINIERI, C. 1970. Madeiras do Parque Estadual do Morro do Diabo. Silvic. São Paulo, São Paulo, 7: $147-150$.

SĀO PAULO. Leis, decretos, etc. 1986. Decreto n 25.342 de 4 de junho de 1986. Diário Oficial, São Paulo, 96(104): 4, 5 de jun. 1986.

SILVA, A.F. 1980. Composição florística e estrutura de um trecho da mata atlântica de Ubatuba (SP). Dissertação de Mestrado. UNICAMP. Campinas.

VENTURA, A.; BERENGUT, G. \& VICTOR, M.A.M. 1965-66. Características edafoclimáticas das dependências do Serviço Florestal do Estado. Silvic. São Paulo, São Paulo, 4/5(4): 57-140. 